\title{
DESIDERATA FOR FUTURE WORK
}

Oort: For the spiral structure in our own Galaxy the most important desideratum would seem to be to get a picture of the spiral arms based on direct distance criteria, independent of the radial velocity. Such a picture might be obtained in the future if a further study of giant $\mathrm{H}$ II regions would provide data for a determination of their distances from optical, infrared or radio observations.

Further extension of the so successful Basel work on distant galactic clusters, possibly by searches in the far infrared, may also contribute in a quite significant way.

A more accurate location of the spiral arms observed in the $21-\mathrm{cm}$ line may be obtained by a large extension of the work on $21-\mathrm{cm}$ absorption lines in the radiation of distant galactic sources. This would appear to be an extremely important undertaking.

Important further information on the dynamics of spiral arms can undoubtedly be obtained from the accurate determination of the birth places of stars by the methods developed by Strömgren. The significance of this was clearly illustrated by the results described by Lin and Yuan during this Symposium.

The greatest advances for the spiral problem in the next few years are likely to come from higher-resolution radio surveys of external galaxies. With large synthesis instruments beamwidths of the order of $\frac{1}{3}$ to $\frac{1}{2} \mathrm{~min}$ of arc will soon be realized, both for continuum observations and for $21-\mathrm{cm}$ line work. For one or two dozens of the nearest large spirals this will make it possible to obtain accurate charts of the arms of neutral hydrogen as well as of those of ionized hydrogen, and of the distribution of the non-thermal radiation relative to the gas arms. It will enable astronomers to obtain fairly reliable data on the relative density of the gas between arms, on the widths of the $\mathrm{HI}_{\mathrm{I}}$ arms and on the relative situation of dust, gas and stars. The line observations will furnish a wealth of data on rotation, on radial components of the gas motion, and on internal motions in the arms. Study of the central regions should permit the investigation of features like the 3-kpc arm in other galaxies, and perhaps to obtain an insight into the relation between such expanding features and the general spiral patterns.

Evidently, more refined multicolour tracings through spiral arms may add greatly to the understanding of their birth process.

It may be desirable in the further development of the theory of spiral structure to take account, on one hand, of the 'events' which apparently cause part of the gas to be carried up to large distances from the galactic plane, and, on the other hand, of the inflow of gas from intergalactic space, which might have sensible effects on the dynamics of the arms. 
Becker: Allow me some words about our future work at the Basel Observatory concerning the galactic disc and the spiral structure.

Although our investigations of galactic clusters and $\mathrm{H}_{\mathrm{II}}$ regions will be continued, they are approaching their natural limits, set by the power of medium size telescopes and by the fact that the photometric determination of distances over $4 \mathrm{kpc}$ is no longer precise enough for reliable results.

Among the roughly thousand galactic clusters in the catalogues of Alter and Rupprecht, some 400 may be found with distances under $4 \mathrm{kpc}$. About 250 have already been observed. Among the remaining 150 clusters, maybe 30 to 40 will be young enough to help to define the spiral pattern according to our experience.

We hope to complete the picture above all in three points. We expect a better definition of the arm following the Perseus arm and we think it might be interesting to know whether the local arm joins really the Scutum-Carina arm in a distance of about 3 to $4 \mathrm{kpc}$ in the direction $l^{\mathrm{II}}=30^{\circ}$ to $55^{\circ}$, as it might be expected according to the radio-astronomical observations of Weaver. The large pitch angle of about $25^{\circ}$ of the local arm is a strong point in favour of this possibility. Finally it would be of considerable interest to find out, whether there exists a connection between the Sagittarius-Scutum arm and the Carina arm in the directions between $300^{\circ}$ and $330^{\circ}$. If this should be the case, the whole feature would have to be called optically ScutumSagittarius-Carina arm.

Unfortunately the number of clusters is not large in the two regions mentioned above. Between $30^{\circ}$ and $55^{\circ}$ there are only 3 , between $300^{\circ}$ and $330^{\circ} 21$ clusters, 7 of which being already observed. Only the observations can decide, whether there are sufficiently young clusters among these objects.

The situation is similar with respect to the H II regions. Optically only those objects are of interest, whose exciting stars are known or can be identified with certainty and localized with optical methods. In the two interesting regions mentioned above, there are 10 and $7 \mathrm{H}$ II regions respectively, the latter ones $\left(l^{\mathrm{II}}=300^{\circ}\right.$ to $\left.330^{\circ}\right)$ being rather small features mostly.

Their exciting stars have not yet been observed, but a corresponding programme might be executed probably in a short time.

Should all these proposed observations of galactic clusters and $\mathrm{H}$ II regions not be able to decide the open questions, then these questions will probably remain unanswered by optical means, since the other indicators of spiral structure cannot be localized individually with sufficient precision.

I want to add some remarks about the possibilities of practical stellar statistics as far as they concern the spiral structure and as far as they are based on photometric measurements. Stars should not be forgotten completely in this context.

Dr. McCuskey has made clear that the important and detailed investigations carried out at the the Warner and Swasey Observatory with stellar-statistical methods of spectral classification and photometry yield no indications of spiral structure in the solar neighbourhood. Their importance lies in another direction.

The gravitational theory of spiral structure leaves no doubt that its findings refer to 
the interstellar matter and to the young objects being born there. However it is not impossible that there exists a certain large scale structure outlined by older stars.

In connection with the problems of spiral structure practical stellar statistics has to take a new attitude. Without any doubt only such a programme can be successful which reaches considerably larger distances than do the spectral classifications. That can be done with a three-colour photometry, which, comprising stars of solar luminosity and brighter than about 20th magnitude, should reach to the limits of the galactic system on our side of its centre. The practically effective limit is set by interstellar absorption in each direction.

A programme covering the local arm and the inter-arm region between the local and the Scutum-Sagittarius arms has been started several years ago in Basel. It is guided by the following consideration: without any doubt there exist discrete star clouds in the Milky Way as well as in other spiral systems. Nor can it be denied that these clouds follow the spiral arms to a large extent.

Since, according to existing results, the stellar clouds do not consist exclusively of interstellar matter and young objects, but also of older stars, the stellar-statistical findings of a three-colour photometry might contribute to a certain extent to the resolution of questions of the spiral structure. In addition to that, one can collect experience concerning the different composition of star clouds regarding the spectral types. This experience might gain some relevance with respect to the connection between spiral structure and evolution. However, it would lead too far in the frame of this symposium, to consider the results of this programme in a more detailed way.

In any case we hope to get additional points of view from the theory of spiral structure, which would allow us to shape the observation programmes in close contact with it.

Lin: I would like to make three short comments from the theoretical point of view.

\section{Mechanism and the Origin of Density Waves}

The observed spiral structure consists of a 'grand design' and many other secondary features. There is presumably a co-existence of material arms and density waves. There may be co-existence of several wave patterns. One of our principal interests in the near future will be the understanding of the mechanisms involved, especially those that can give rise to the wave patterns.

From our experience with plasma physics, we know that there is a variety of mechanisms for instabilities in plasmas. We must therefore not close our eyes to the possibility of several mechanisms giving rise to similar spiral patterns. We must, however, guard against invoking physically non-existent components as a cause for instability. Personally, I am still convinced of the importance of gravitational instability in the outer reaches of the galaxies, as I described in my talk.

As I also mentioned there, attention should be directed to resonant stars that play an important role in the mechanism of maintaining density waves in stellar systems. 
We wish to associate the ring structure in external galaxies and the 3-kpc arm in the Milky Way with Lindblad resonance.

\section{Application to External Galaxies}

Besides continuing to push for better comparison between theory and observations within our own Galaxy, we should now turn our attention to external galaxies. There are at least three lines of research that one could pursue.

(a) Normal spirals: determination of the pattern. After the rotation curve and the mass model are established for an external galaxy, one can get a first approximation to its spiral pattern, if the pattern speed is known. We now have a way of estimating this approximately, since the outer edge of the spiral pattern is roughly associated with co-rotation. It is my understanding that Frank $\mathrm{H}$. Shu is pursuing this approach in connection with the galaxy M51.

(b) Barred spirals: a conjecture. The arrangement of dust lanes and prominent $\mathrm{H}$ II regions in a galaxy such as NGC 1300 suggest that they might also be caused by gas undergoing a sudden compression while flowing through a density wave pattern. Such a picture would also be compatible with the dust lanes along the bar. We therefore propose the re-examination of the theory of barred galaxies from this point of view. This view is compatible with the gradual transition between normal spirals and barred spirals, as strongly advocated by de Vaucouleurs.

(c) Ring structure: high resolution observation in external galaxies. We know that the 3-kpc arm separates the part of the galaxy abundaint with Hil regions from that deficient in $\mathrm{H}$ II regions, and we believe that there is a ring structure similar to that seen in external galaxies, such as NGC 5364. I would therefore like to recommend the careful examination of the behaviour of the gas and stars near the ring of NGC 5364 for possible anomalous behaviour similar to that associated with the 3-kpc arm. Consultation with Van den Bergh indicates that the optical observations needed are within the realm of observational possibilities. For the observation of neutral hydrogen, much higher resolution is needed than that available at the present time.

\section{Physical Processes in the Interstellar Medium}

Having gone this far with the understanding of the grand design, we can turn our attention to the next smaller scale, to consider the rolling motion (torsional oscillation?) of a spiral arm and its implications on the magnetic field, as discussed by Woltjer. We have noted that we can account for the wider separation between the dust lane and the HII arm in the inner part of M5I (and the narrower separation in the outer parts), as emphasized by Morgan. In general, we can look for a deeper understanding of the physical processes in the interstellar medium once we know there are density waves.

Since the several spiral arms nearby are presumably not all of the same nature, a comparative study of the physical processes in them would be very helpful to our understanding. Strong compression due to density waves triggers the formation of bright stars and $\mathrm{H}_{\mathrm{II}}$ regions. This same dynamical process would have its effect on 
Hi regions, on the magnetic field (and its consequent effect on cosmic ray particles), on the formation of dust lanes, and possibly on the formation of molecules as well. We can indeed look forward to an exciting time in the study of interstellar medium.

\section{Discussion}

Van den Bergh: It is the purpose of this remark to draw attention to a rather striking feature of spiral structure in galaxies that has not been discussed at this Symposium. Observations of late-type galaxies show that the strength of the spiral phenomenon is a function of the luminosity (or mass) of the galaxy in which it occurs. Supergiant galaxies tend to have long and well developed spiral structure. Normal giant galaxies usually exhibit only rudimentary spiral structure. Finally the majority of late-type dwarf galaxies exhibit no spiral structure at all, i.e. they are Magellanic type irregulars. The dependence of the ratio of spirals to irregulars on luminosity is illustrated by the data given in Table I.

\section{TABLE I}

Percentage of all intermediate- and late-type galaxies which are irregular $\left(H=100 \mathrm{~km} \mathrm{~s}^{-1} \mathrm{Mpc}^{-1}\right.$ assumed $)$

\begin{tabular}{lrl}
\hline$\left\langle M_{\mathrm{pv}}\right\rangle$ & No. galaxies classified & $\begin{array}{l}\text { Per cent } \\
\text { irregular }\end{array}$ \\
& & \\
-20.1 & 113 & 0 \\
-19.2 & 201 & 0.5 \\
-18.2 & 93 & 7.5 \\
-16.6 & 88 & 36 \\
$-15.0:$ & 104 & 62 \\
\hline
\end{tabular}

